\title{
The intrinsic emission of Seyfert galaxies observed with BeppoSAX/PDS
}

\section{Comparison of the average spectra of the three classes of Seyfert galaxies}

\author{
S. Deluit ${ }^{1,2}$ and T. J.-L. Courvoisier ${ }^{1,2}$ \\ 1 INTEGRAL Science Data Center, 16 Chemin d'Ecogia, 1290 Versoix, Switzerland \\ 2 Geneva Observatory, 51 Chemin des Maillettes, 1290 Sauverny, Switzerland
}

Received 30 April 2002 / Accepted 27 November 2002

\begin{abstract}
We present a study of the hard X-ray spectrum $(>15 \mathrm{keV})$ of different classes of Seyfert galaxies observed with BeppoSAX/PDS. Using hard X-ray data, we avoid absorption effects modifying the Seyfert emission and have direct access to the central engine of these sources. The aim of this study is first to characterize the general properties of the hard X-ray spectrum of Seyfert 1, 1.5 and 2 galaxies and secondly to compare their intrinsic emission to test unified models according to which all the classes have the same nucleus.

We compute the average spectrum of 14 Sy 1,9 Sy 1.5 and 22 Sy 2 galaxies observed by the PDS (15-136 keV). The average spectrum of Sy 1 differs from that of Sy 2, the first requiring the presence of a high energy cutoff which is absent in the second. We also show that the reflection component is possibly more important in the Sy 2 emission. The nature of Sy 1.5 galaxies is ambiguous as they have a negligible reflection component (like Sy 1) and do not require a cutoff (like Sy 2).
\end{abstract}

Key words. galaxies: active - galaxies: nuclei - galaxies: Seyfert - gamma rays: observations - methods: data analysis methods: statistical

\section{Introduction}

According to unified models (Antonucci \& Miller 1985), Seyfert 2 galaxies harbor a bright Seyfert 1 nucleus which is hidden from our view by an optically and geometrically thick obscuring torus. Consequently, the difference between type 1 and 2 Seyfert galaxies would be only due to viewing angle. Several observational facts in favor of unified models have been found:

- detection of Polarized Broad Lines (PBL hereafter) in several Seyfert 2 galaxies (Antonucci \& Miller 1985; Miller \& Goodrich 1990; Tran 1995; Young et al. 1996; Awaki et al. 2000; Moran et al. 2000; Alexander 2001; Gu et al. 2001; Lumsden et al. 2001), interpreted as scattering of the Broad Lines Region emission by warm material placed above an absorption torus;

- large absorbing column densities observed in the hard Xray spectra of Seyfert 2 galaxies (Guainazzi et al. 2001 and references therein);

- suspicion of anisotropic ionization radiation from the nucleus through the structures in the light of [OIII] line at 5007 A (e.g. in NGC 5252, Tadhunter \& Tsvetanov 1989).

$\mathrm{X}$-ray data have played a major role in support of unified models. The X-ray spectra of Seyfert galaxies are known relatively

Send offprint requests to: $\mathrm{S}$. Deluit,

e-mail: Sandrine.Deluit@obs.unige.ch well and are composed at energies above $3 \mathrm{keV}$ of a power law, a Fe line and a Compton reflection component (Nandra \& Pounds 1994; Gondek et al. 1996; Nandra et al. 1997; Zdziarski et al. 1999, 2000). Some questions remain like the shape and the presence of a cutoff at higher energies. Furthermore, some observations are inconsistent with unified models at least in their simplest formulation. High energy spectra of a sample of Seyfert 2 galaxies observed by OSSE suggest an intrinsic difference in the hard X-ray emission of both classes of Seyfert galaxies. The two classes differ in the slope of the intrinsic power law, in the energy of the exponential cutoff, in the amount of absorption and reflection (Zdziarski 1995).

Hard X-ray spectra are a powerful tool to probe and test unification models by providing a direct view on the central engine emission mostly free of absorption effects. The form of the individual hard X-ray spectra is poorly constrained due to limited photon statistics. This can be improved by considering the average spectrum of a sample to extract the properties of a class.

We study the hard X-ray emission (above $15 \mathrm{keV}$ ) of Seyfert $1,1.5$ and 2 galaxies in order to investigate several questions:

- Are the high energy index slope of Seyfert 2 and Seyfert 1 identical?

- Do the Seyfert 1 and 2 spectra have a cutoff? In thermal Compton models the high energy cutoff provides a 
reliable estimate of the comptonizing material temperature (Rybicki \& Lightman 1979). Therefore, differences in cutoffs or their absence indicate different comptonizing medium temperatures.

- What is the importance of Compton reprocessed emission for each class? Could the reflection be the only cause to the observed difference between Seyfert 1 and Seyfert 2?

To answer these questions, we compute the average PDS spectrum of each class. We consider for inclusion in the Seyfert 2 sample only galaxies for which the absorption column density is less than $7 \times 10^{23} \mathrm{~cm}^{-2}$ to ensure that, above $15 \mathrm{keV}$, absorption is less than $50 \%$ of the flux. We are thus in the position to characterize the intrinsic emission of the various types of objects.

\section{Selection procedure of our sample}

We start by including all (i.e. 68) Seyfert PDS spectra available in the public archive of BeppoSAX Data Center ${ }^{1}$. We have investigated the possibility of source confusion in the PDS aperture by looking in the MECS images and excluded two confused objects from this study. We then form three subsamples characterizing Sy 1, Sy 1.5 and Sy 2 galaxies respectively.

\subsection{The nature of objects composing the subsamples}

We follow the optical classification of Seyfert galaxies indicated in the NASA/IPAC Extragalactic Database ${ }^{2}$ (NED) and exclude LINERs for the ambiguity of their classification. We include Sy 1 and Sy 1.2 galaxies in the Seyfert 1 subsample, Sy 1.5 in the Seyfert 1.5 subsample and Sy 1.9 and Sy 2 in the Seyfert 2 subsample. We exclude Seyfert 1.8 for which the classification is too ambiguous between Seyfert 1.5 and Seyfert 2 galaxies.

\subsection{Absorption effects in Seyfert 2 galaxies emission}

Hard X-rays give a direct access to the intrinsic emission provided that we avoid absorption effects which contaminate the Seyfert 2 galaxies spectra at lower energies. Objects with $N_{\mathrm{H}}>$ $1.5 \times 10^{24} \mathrm{~cm}^{-2}$ (so called Compton thick) have a Thomson optical depth larger than one. Electron scattering modifies their emission even in the hard X-ray energy domain. We therefore eliminated all such objects from consideration. In summary, we consider only objects for which $\mathrm{e}^{-\sigma_{\mathrm{T}} N_{\mathrm{H}}} \times \mathrm{e}^{-\sigma_{\mathrm{ph}}(E) N_{\mathrm{H}}}$ is larger than 0.5 at $15 \mathrm{keV}, \sigma_{\mathrm{T}}$ and $\sigma_{\mathrm{ph}}$ are respectively the Thomson and the photoelectric cross section. This means that we keep only objects having a column density less than $7 \times 10^{23} \mathrm{~cm}^{-2}$.

The role of absorption in our Seyfert 2 class study is exhaustively explained in the Appendix B.

We present in Fig. 1 the column density distribution for the Seyfert 2 galaxies sample. The majority of the objects have $N_{\mathrm{H}}$ between $10^{22} \mathrm{~cm}^{-2}$ and $10^{23} \mathrm{~cm}^{-2}$.

We present in Fig. 2 the hydrogen column density distribution versus the redshift for Seyfert 2 galaxies.

\footnotetext{
1 http://www .asdc.asi.it/bepposax/

2 http://nedwww.ipac.caltech.edu/
}

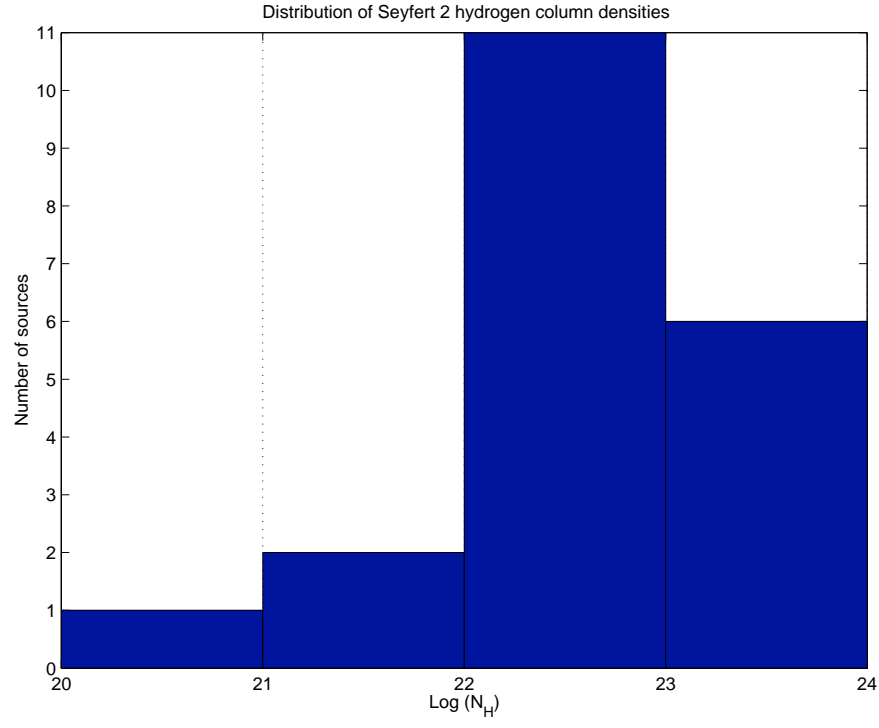

Fig. 1. Distribution of the hydrogen column density of sources composing our Seyfert 2 sample.

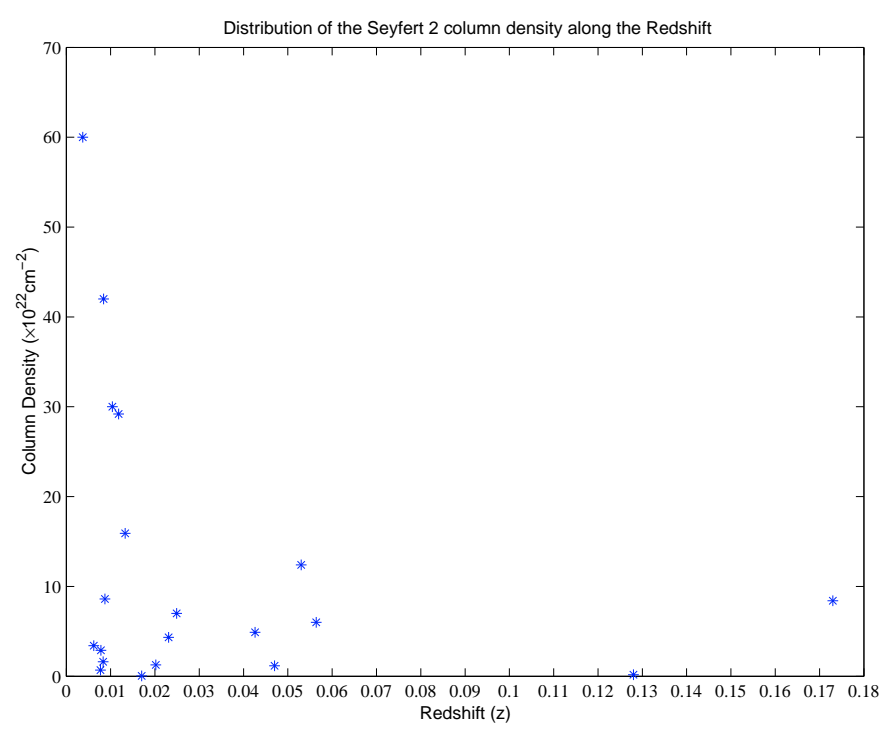

Fig. 2. Distribution of Seyfert 2 hydrogen column density along the redshift.

We note a large number of high column densities at low redshift. This is probably an observational artefact due to the fact that distant and highly absorbed Seyfert 2 galaxies are hardly detectable.

\subsection{Data quality criterion}

The third criterion we introduce is the quality of the data. We calculate the integrated signal to noise ratio of each object in the PDS energy range (15-136 keV). The data are used only when the integrated signal to noise ratio is equal to or larger than $2 \sigma$. This threshold is high enough to conclude to a detection by the PDS detector for a known source. 

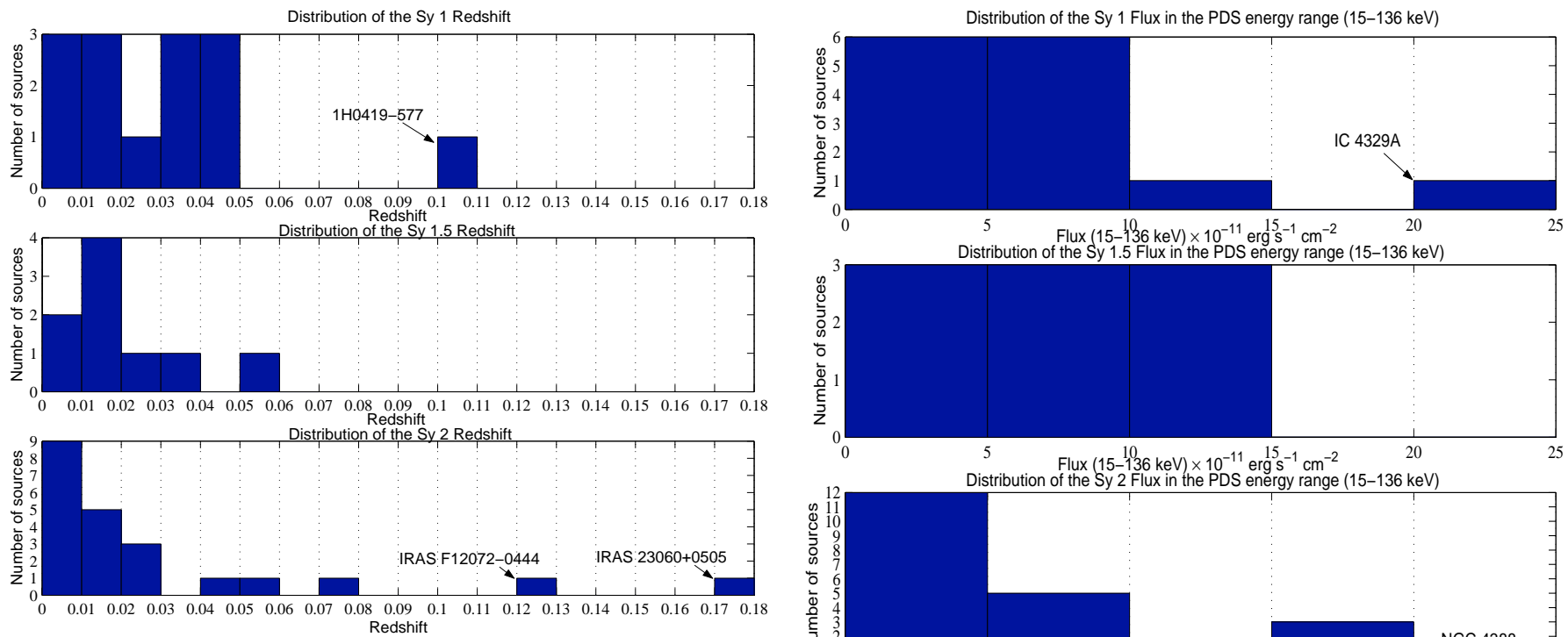

Fig. 3. Redshift distribution for the three classes of Seyfert galaxies.
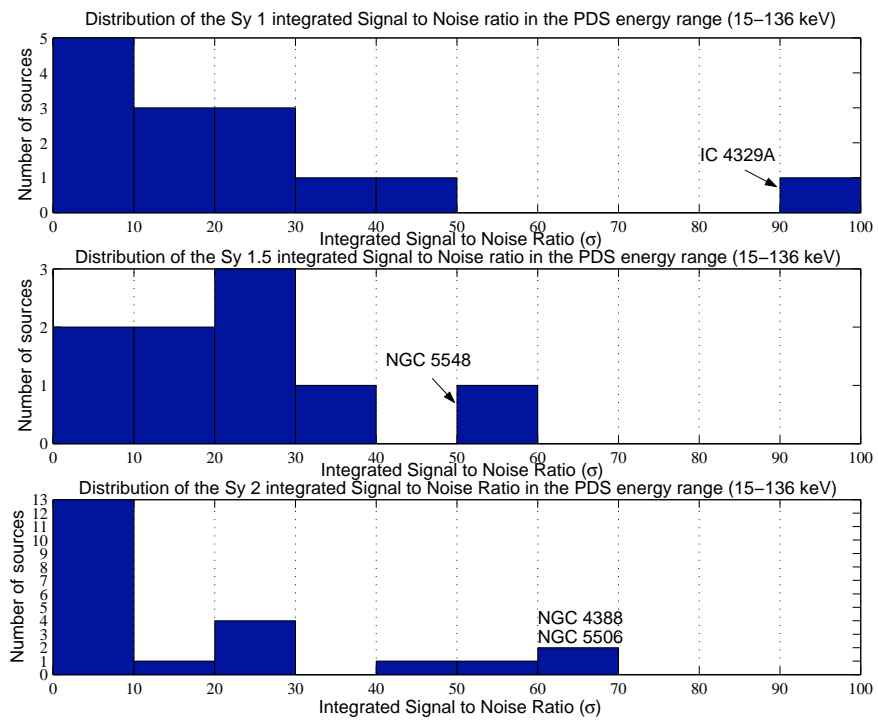

Fig. 4. Distribution of the integrated signal to noise ratio for the three classes of Seyfert in the PDS energy range.

\section{Properties of our samples}

\subsection{Redshift distributions}

We present in Fig. 3 the redshift distribution for the three classes of Seyfert galaxies.

Most of the objects have a redshift less than 0.05 (93\% for Sy $1,89 \%$ for Sy 1.5 and $82 \%$ for Sy 2 galaxies). The difference between the redshift distributions of Sy 1 and Sy 2 galaxies may be due to biases in object selections when planning X-ray observation programmes. Indeed, absorbed objects being more difficult to observe will be looked for at small distances.

\subsection{Comparison of the data quality}

We determine the integrated signal to noise ratio in the PDS energy range (15-136 keV) for each object (Fig. 4).

The majority of the objects have an integrated signal to noise ratio less than $30 \sigma$ (79\% for Sy 1, 78\% for Sy 1.5 and $82 \%$ for Sy 2 galaxies). The Seyfert 1, 1.5 and 2 samples are dominated by low signal to noise spectra.

\subsection{Flux distributions}

We measure the integrated flux in the PDS energy range of each object (Fig. 5). The majority of the sources have a hard $\mathrm{X}$-ray flux less than $15 \times 10^{-11} \mathrm{erg} \mathrm{s}^{-1} \mathrm{~cm}^{-2}$ between $15 \mathrm{keV}$ and $136 \mathrm{keV}$.

The proportion of objects with a flux less than or equal to $15 \times 10^{-11} \mathrm{erg} \mathrm{s}^{-1} \mathrm{~cm}^{-2}$ is of $93 \%$ for Sy $1,100 \%$ for Sy 1.5 and of $82 \%$ for Sy 2 galaxies.

\subsection{Luminosity distributions}

From the flux we calculate the integrated luminosity using $H_{0}=75 \mathrm{~km} \mathrm{~s}^{-1} \mathrm{Mpc}^{-1}$ and $q_{0}=0.5$ as cosmological parameters. The results are shown in Fig. 6.

The majority of the objects have a hard X-ray luminosity less than or equal to $30 \times 10^{43} \mathrm{erg} \mathrm{s}^{-1}$ between $15 \mathrm{keV}$ and $136 \mathrm{keV}$ (93\% for Sy 1, 100\% for Sy 1.5 and $91 \%$ for Sy 2). In the Seyfert 2 subsample, two objects have a luminosity above $30 \times 10^{43} \mathrm{erg} \mathrm{s}^{-1}$ : IRAS F12072-0444 with a luminosity of $61.5 \times 10^{43} \mathrm{erg} \mathrm{s}^{-1}$ and IRAS $23060+0505$, with a luminosity of $111 \times 10^{43} \mathrm{erg} \mathrm{s}^{-1}$. These two galaxies are warm Ultra Luminous Infrared Galaxies and are probably quasars regarding their hard X-ray luminosity. However, they are classified as Seyfert 2 galaxies in NED and therefore kept in our sample. 

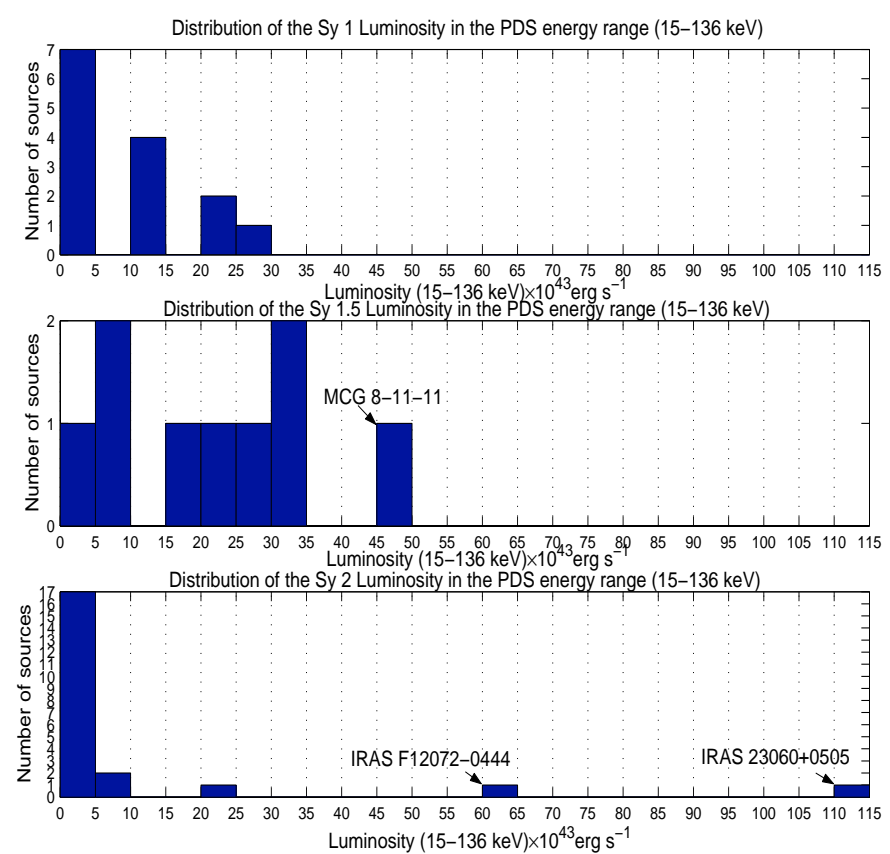

Fig. 6. Luminosity distribution for the three classes of Seyfert in the PDS energy range.

\subsection{Excluded objects}

Several objects have been excluded from our initial sample. We review below all the dismissed sources and the reason of their exclusion.

1. NGC 4151: with an integrated signal to noise ratio of $227.5 \sigma$ and an integrated flux in the PDS energy range of $58 \times 10^{-11} \mathrm{erg} \mathrm{s}^{-1} \mathrm{~cm}^{-2}$, this object would strongly dominate the sample. We will discuss this bright Seyfert 1.5 galaxy in the Paper II of this study where individual objects are discussed (Deluit \& Courvoisier 2003).

2. Mrk 1073: this object is not detected in the MECS and LECS instruments and has a very high signal in the PDS. We excluded it from our sample because its PDS spectrum is probably in part due to a nearby source.

3. NGC 1386: a large fraction of the PDS flux is due to the nearby NGC 1365 (Maiolino et al. 1998).

4. MCG 5-18-002: we excluded this source in reason of its uncertain absorption given as $N_{\mathrm{H}}>10^{25} \mathrm{~cm}^{-2}$ by Maiolino et al. (1998). The value is based only on the [OIII] flux and requires further confirmation.

5. Considering the data quality criterion, we also exclude four Seyfert 1 (TON S180, GQCOMAE, IRAS 132243809 and Mrk 478) and four Seyfert 2 (NGC 3081, IRAS 11058-1131, IRAS 10126+7339 and NGC 4941). All these objects have an integrated signal to noise ratio less than $2 \sigma$.

\subsection{The final sample}

The initial sample of all Seyfert galaxies observed with BeppoSAX included 68 objects among 18 Sy 1,10 Sy 1.5 and 40 Sy 2 galaxies.
Our sample is finally composed of 67 observations for a total of 45 Seyfert galaxies of which 14 are Seyfert 1 galaxies, 9 are Seyfert 1.5 and 22 are Seyfert 2 galaxies.

The list of objects composing our subsamples, their characteristics and their spectral properties is given in Table A.1 for Sy 1 and Sy 1.5 galaxies samples and in Table A.2 for the Sy 2 galaxies sample.

\section{Data analysis}

The BeppoSAX instruments cover a wide spectral band and consist of four co-aligned Narrow Field Instruments (NFI) plus two Wide Field Cameras perpendicular to the axes of the NFI and looking in opposite directions.

The NFI is composed by a Low Energy Concentrator Spectrometer (LECS: Parmar et al. 1997), three Medium Energy Concentrator Spectrometer (MECS: Boella et al. 1997) and a Phoswich Detector Counter (PDS: Frontera et al. 1997). LECS and MECS have imaging capabilities and operate respectively in the $0.1-5 \mathrm{keV}$ and $2.0-10 \mathrm{keV}$. The instrument used in this study, the PDS, is an instrument using rocking collimators, the energy band is between 15-200 keV. The PDS consists in four phoswich units operating in collimator mode with two of them pointing towards the source, the other two away. The two pairs switch on and off source and the net source spectra are obtained by subtracting the "off" (which represent the background) from the "on" counts. The PDS data available on the web are already background subtracted. A more detailed description of the background and of the subtraction procedure is available from http://www.sdc.asi.it/software.

The data analysis has been performed with the XSPEC (Arnaud 1996) version 11 package and using the last PDS response matrices released by the SAX Data Center.

We kept in the input and output files the same binning for the average spectrum as was available for the original data of each source. For a source observed several times, we first compute its average spectrum. We then compute the average subclass spectrum taking into account of the exposure time of each data set. The energy band is from 15 to $136 \mathrm{keV}$.

\section{Spectral properties of the different classes of Seyfert galaxies}

We present in Fig. 7 the average counts spectrum of the three classes of Seyfert galaxies.

The spectrum of each class has an integrated signal to noise ratio of $96 \sigma$ for Seyfert 1 galaxies, $74.4 \sigma$ for Seyfert 1.5 galaxies and $94 \sigma$ for Seyfert 2 galaxies. No normalization has been applied to obtain the average spectra shown in this figure.

We compare the Seyfert classes following two ways. First through a model independent method comparing directly the counts spectra. Secondly through a model dependent method using a fitting procedure to investigate the physical processes at the origin of the emission. 


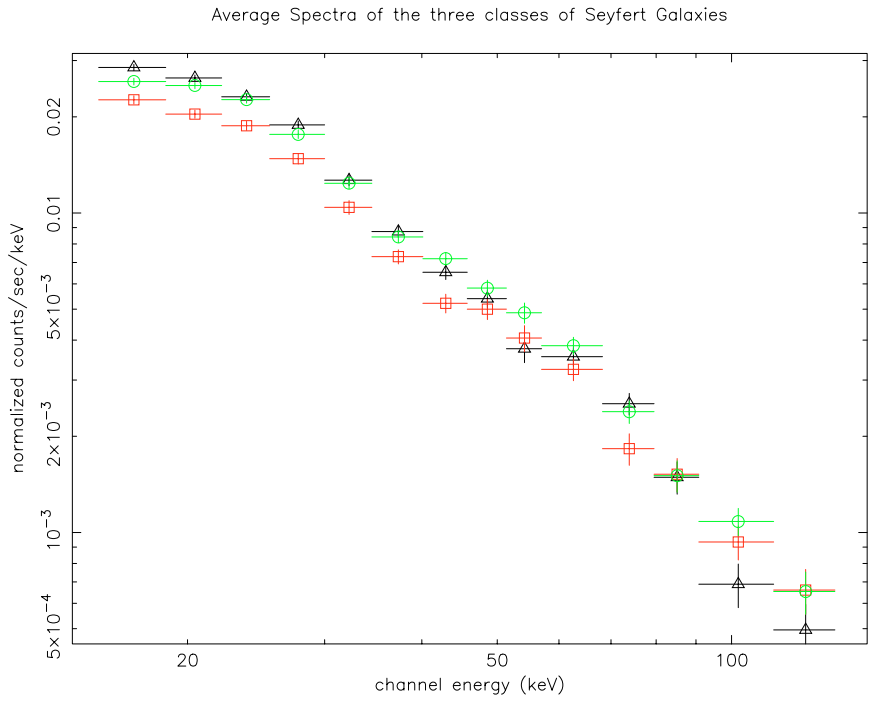

Fig. 7. Average spectra of the three classes of Seyfert galaxies: Sy 1 (triangle), Sy 1.5 (square) and Sy 2 galaxies (circle).

\subsection{Model independent method: Comparison of the normalized counts spectra}

We compare the different average spectra normalizing them at $15 \mathrm{keV}$. This allows us to compare their emission independently of the average flux. The result is shown in Fig. 8. On the left column, we present the normalized counts spectra of the three pairs of classes, on the right side we give the ratio of each pair.

\subsubsection{Comparison between Seyfert 1 and Seyfert 2 emission}

The two spectra are similar below $40 \mathrm{keV}$ confirming that the Sy 2 galaxies emission is not significantly modified by absorption effects. Above $40 \mathrm{keV}$, the Sy 2 emission dominates that of Sy 1 . The difference between the two classes increases above $90 \mathrm{keV}$. Indeed, the hard photons population is more important in Sy 2 than in Sy 1 galaxies showing that the observed Sy 2 spectrum is harder than Sy 1. The deficit of hard photons in the Sy 1 class can be explained either by the presence of a cutoff in its emission and/or by a reflection process occurring in the Sy 2 emission. The possibility that the Sy 2 intrinsic power law is harder than Sy 1 cannot be excluded. The Sy 1 average emission is $42 \%$ weaker than that of Sy 2 around $100 \mathrm{keV}$ where they differ most in the normalized ratio.

\subsubsection{Comparison between Seyfert 1 and Seyfert 1.5 emission}

The spectra are identical below $40 \mathrm{keV}$. The Sy 1.5 emission slightly dominates between 40 and $65 \mathrm{keV}$. At higher energies, the Sy 1.5 spectrum contains a more important hard photons population. As in Sy 2 galaxies, the Sy 1.5 emission domination above $80 \mathrm{keV}$ can be produced by a cutoff in Sy 1 or by a harder Sy 1.5 primary power law. The Sy 1 average emission is $41 \%$ weaker than that of Sy 1.5 at $\sim 100 \mathrm{keV}$ where they differ most in the normalized ratio.

\subsubsection{Comparison between Seyfert 1.5 and Seyfert 2 emission}

The two spectra remain very close along the PDS energy band. Indeed, the maximal deviation between the two spectra in the normalized ratio does not exceed $20 \%$ at $\sim 40 \mathrm{keV}$ where the Sy 2 average emission is marginally higher than that of Sy 1.5. Thus, we consider in a first approach that the spectrum of Sy 1.5 and Sy 2 present notable similarities.

\subsubsection{Summary}

The main result of this model independent study is that Sy 2 differ from Sy 1 galaxies and that Sy 1.5 and Sy 2 are similar. In particular in the high energy domain where the Sy 1.5 and Sy 2 classes present a more important hard photons population compared with Sy 1 galaxies. The processes that can be responsible for the observed differences between the three classes is investigated in the next subsection.

\subsection{Model dependent method: Investigating the physical processes into Seyfert galaxies engine}

To interpret our data in terms of models taking the PDS spectral response matrices into account, we fit the spectra with different theoretical models. We first fit the spectra with simple models (power law, broken power law and cutoff power law). We then consider reflection with the PEXRAV model described below.

All the results are summarized in Tables 1 and 2 .

\subsubsection{Fitting of the spectra with simple theoretical models}

The power law model gives the worst statistical results for all the classes. For Sy 1 and 2 galaxies, this model is statistically unacceptable with a reduced $\chi^{2}$ greater than 2 indicating that their spectra require an additional component. For the Sy 1.5 galaxies, this model is more adapted.

Sy 1 emission requires a cutoff considering both the statistical result of the $\chi^{2}$ test and the FTEST probability, confirming our model independent analysis. The presence of a cutoff is unprobable in Sy 1.5 and Sy 2 galaxies. Indeed, the $\chi^{2}$ method gives the best result for a broken power law $\left(\chi_{\mathrm{r}}^{2}=1.03\right.$ for Sy 1.5 and $\chi_{\mathrm{r}}^{2}=1.30$ for Sy 2 ) compared with the cutoff model $\left(\chi_{\mathrm{r}}^{2}=1.21\right.$ and $\chi_{\mathrm{r}}^{2}=1.60$ respectively).

In Sect. 5.1, we noted two maxima in the ratio between Sy 1 and Sy 2 spectra around $40 \mathrm{keV}$ and $100 \mathrm{keV}$. These features may be due to a combination of a cutoff in Sy 1 (here found at $70_{-17}^{+30} \mathrm{keV}$ ) and/or a reflection hump in Sy 2 class that we investigate in the next section.

\subsubsection{Fitting of the spectra with the reflection model PEXRAV}

Previously, we suspected the presence of a reflection component in the Sy 1.5 and Sy 2 spectrum. We investigate this 

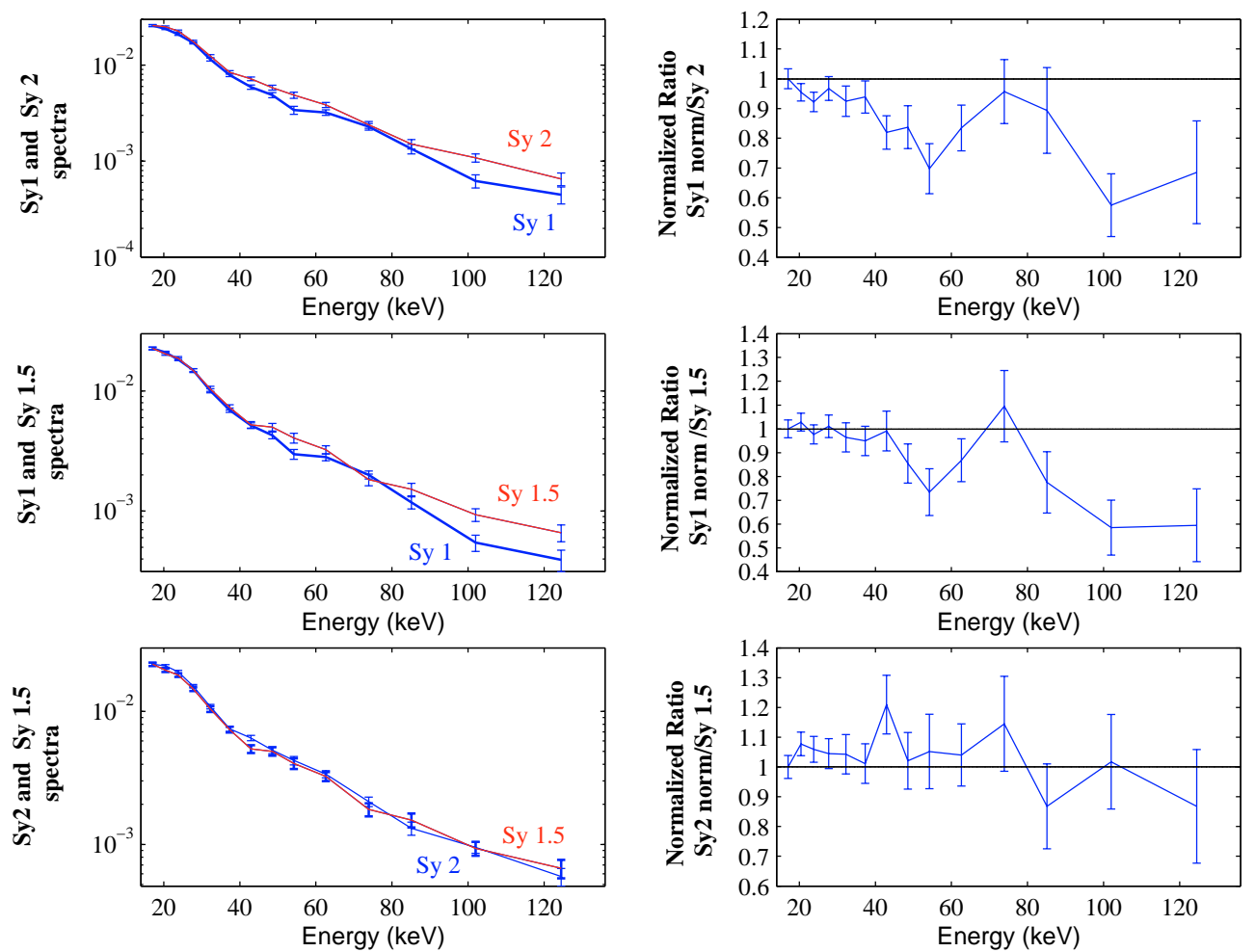

Fig. 8. Left: three pairs of Spectral Energy Distributions normalized at $15 \mathrm{keV}$. Right: ratios of these SEDs.

Table 1. Best fit parameters for simple models.

\begin{tabular}{|c|c|c|c|c|c|c|c|}
\hline Model & $\begin{array}{l}\text { Seyfert } \\
\text { Class }\end{array}$ & $\begin{array}{c}\Gamma \\
\text { Index Slope }\end{array}$ & $\begin{array}{c}E_{\text {break/cutoff }} \\
(\mathrm{keV})\end{array}$ & $\begin{array}{c}\Gamma_{2} \\
\text { Index Slope }\end{array}$ & $\chi^{2} /$ d.o.f. & $\begin{array}{c}\chi_{\mathrm{r}}^{2} \\
\text { reduced }\end{array}$ & $\begin{array}{c}\text { FTEST } \\
\text { Probability value }\end{array}$ \\
\hline \multirow[t]{3}{*}{ Power Law } & 1 & 2.03 & - & - & $50.0 / 12$ & 4.17 & - \\
\hline & 1.5 & $1.90_{-0.05}^{+0.05}$ & - & - & $16.7 / 12$ & 1.39 & - \\
\hline & 2 & 1.90 & - & - & $44.2 / 12$ & 3.68 & - \\
\hline \multirow[t]{3}{*}{ Broken Power Law } & 1 & $1.65_{-0.21}^{+0.16}$ & $28_{-3.8}^{+7.0}$ & $2.25_{-0.09}^{+0.12}$ & $14.7 / 10$ & 1.47 & $2.2 \times 10^{-3}$ \\
\hline & 1.5 & $1.62_{-0.38}^{+0.21}$ & $26_{-5.4}^{+16}$ & $2.01_{-0.09}^{+0.13}$ & $10.3 / 10$ & 1.03 & $5.0 \times 10^{-2}$ \\
\hline & 2 & $1.36_{-0.56}^{+0.24}$ & $24_{-2.1}^{+4.3}$ & $2.04_{-0.06}^{+0.07}$ & $13.9 / 10$ & 1.39 & $3.1 \times 10^{-3}$ \\
\hline \multirow[t]{3}{*}{ Cutoff Power Law } & 1 & $1.49_{-0.17}^{+0.17}$ & $70_{-17}^{+30}$ & - & $15.2 / 11$ & 1.38 & $4.5 \times 10^{-4}$ \\
\hline & 1.5 & $1.64_{-0.19}^{+0.11}$ & $152_{-66}^{+48}$ & - & $13.3 / 11$ & 1.21 & $5.8 \times 10^{-2}$ \\
\hline & 2 & $1.45_{-0.17}^{+0.15}$ & $87_{-24}^{+42}$ & - & $17.6 / 11$ & 1.60 & $1.8 \times 10^{-3}$ \\
\hline
\end{tabular}

Note: The uncertainties correspond to $90 \%$ confidence level based on a $\Delta \chi^{2}=2.7$ criterion (Lampton et al. 1976).

hypothesis using the PEXRAV model (Magdziarz \& Zdziarski $1995)$ in XSPEC. This model calculates the expected X-ray spectrum when a point source of X-rays is incident on optically thick, mainly neutral material. The parameter $R$ measures the reflection component. If the primary source is isotropic and neither the source nor the reflector are obscured, then $R$ can be linked to the solid angle seen through the reflector as $R \simeq \frac{\Omega}{2 \pi}$. This model and in particular the reflection component are inclination dependent. We apply this model in two ways: first, we fix the inclination from the normal of the disk at a medium angle to test the occurrence of the reflection as well as the presence of other components independently to any strong inclination influence. We then change the angle value fixing or letting it free in order to understand its role and to obtain the inclination value for which we have the best fit. The results are shown in Table 2.

1. Intermediate inclination analysis

The inclination is initially fixed at an intermediate value of $\cos \theta=0.45$.

The $\chi^{2}$ test indicates that PEXRAV significantly improves the data fitting of Sy 1 and Sy 2 galaxies compared with simple models, not however for the Sy 1.5 spectrum that remains best represented by a broken power law. Therefore, reflection may play a role for the two main Seyfert classes and be absent in Sy 1.5 emission. This is confirmed by the reflection amount which can be non existent for Sy $1.5(R=$ $\left.0.73_{-0.71}\right)$ whereas its presence is strongly suggested in Sy 2 emission $\left(R=1.47_{-0.77}\right)$ at the $2 \sigma$ level. The primary power 
Table 2. Best fit parameters for the reflection model PEXRAV.

\begin{tabular}{ccccccc}
\hline \hline \multicolumn{7}{c}{ PEXRAV PARAMETERS } \\
\hline $\begin{array}{c}\text { Seyfert } \\
\text { Class }\end{array}$ & $\begin{array}{c}\Gamma \\
\text { Index Slope }\end{array}$ & $\begin{array}{c}\text { Efolded } \\
(\mathrm{keV})\end{array}$ & $\begin{array}{c}R \\
\text { Reflection }\end{array}$ & $\chi^{2} /$ d.o.f. & $\begin{array}{c}\chi_{\mathrm{r}}^{2} \\
\text { reduced }\end{array}$ & $\begin{array}{c}\cos \theta \\
\text { fixed }\end{array}$ \\
\hline 1 & $1.92_{-0.49}^{+0.26}$ & $238_{-176}^{+N C}$ & $0.93_{-\mathrm{NC}}^{+2.00}$ & $13.2 / 10$ & 1.32 & 0.45 \\
1.5 & $1.96_{-0.06}^{+0.06}$ & $9.8 \cdot 10_{[a]}^{5}$ & $0.73_{-0.71}^{+0.81}$ & $10.8 / 10$ & 1.08 & 0.45 \\
2 & $1.99_{-0.45}^{+0.05}$ & $2.4 \cdot 10_{[a]}^{4}$ & $1.47_{-0.77}^{+1.07}$ & $13.1 / 10$ & 1.31 & 0.45 \\
\hline 1 & $1.89_{-0.46}^{+0.28}$ & $221_{-158}^{+N C}$ & $0.48_{-\mathrm{NC}}^{+1.54}$ & $13.1 / 10$ & 1.31 & 0.87 \\
1.5 & $1.95_{-0.05}^{+0.06}$ & $8.2 \cdot 10_{[a]}^{5}$ & $0.39_{-0.26}^{+0.38}$ & $10.7 / 10$ & 1.07 & 0.87 \\
2 & $2.00_{-0.45}^{+0.05}$ & $3.6 \cdot 10_{[a]}^{4}$ & $2.33_{-0.49}^{+1.96}$ & $13.0 / 10$ & 1.30 & 0.30 \\
\hline Seyfert & $\Gamma$ & Efolded & $R$ & $\chi^{2} /$ d.o.f. & $\chi_{\mathrm{r}}^{2}$ & $\cos \theta$ \\
Class & Index Slope & $(\mathrm{keV})$ & Reflection & & reduced & free \\
\hline 1 & $1.91_{-0.47}^{+0.29}$ & $231_{-168}^{+N C}$ & $0.53_{-0.27}^{+N C}$ & $13.2 / 9$ & 1.47 & 0.81 \\
1.5 & $1.95_{-0.09}^{+0.06}$ & $1.0 \cdot 10_{[a]}^{6}$ & $0.36_{-0.23}^{+N C}$ & $10.7 / 9$ & 1.19 & 0.95 \\
2 & $2.00_{-0.44}^{+0.05}$ & $2.8 \cdot 10_{[a]}^{3}$ & $2.55_{-2.13}^{+N C}$ & $13.0 / 9$ & 1.44 & 0.28 \\
\hline \multicolumn{7}{c}{} \\
\hline
\end{tabular}

Notes:

The uncertainties correspond to $90 \%$ confidence level based on a $\Delta \chi^{2}=2.7$ criterion (Lampton et al. 1976).

$\mathrm{NC}$ indicates that no constraint has been found on this limit.

$[a]$ the value indicates that the high energy cutoff is far above values that can be measured by the PDS showing the absence of a measured cutoff.

law indices of the three classes are within $1 \sigma$, however Sy 2 emission could be softer compared with the Sy 1 index slope $\left(\Gamma=1.99_{-0.45}^{+0.05}\right.$ for Sy 2 against $\Gamma=1.92_{-0.49}^{+0.26}$ for Sy 1$)$. A strong difference appears in the high energy cutoff which is relatively well identified for Sy $1\left(E_{\mathrm{c}}=238_{-176} \mathrm{keV}\right)$ and totally absent for Sy 1.5 and Sy 2 classes. The main result is that the spectrum of Sy 2 galaxies differs from that of Sy 1 with the absence of a cutoff and a possible presence of an important reflection component in the Sy 2 emission.

2. Inclination influence

We change the inclination value to test unified models which suggest that Sy 2 galaxies are seen edge-on.

(a) Fixed inclination at different angles

We fix the inclination at $\cos \theta=0.30$ and $\cos \theta=0.87$.

The influence of the inclination parameter is not crucial on the final result of the $\chi^{2}$ test. The best fit for Sy 1 and Sy 1.5 is obtained for $\cos \theta=0.87$, an angle of $30^{\circ}$ from the normal of the disk. The reflection component value is lower for $\cos \theta=0.87$ than for an inclination of $\cos$ $\theta=0.45$ as predicted by the fact that the reflection is inclination dependent in the PEXRAV model. We also confirm that we obtain for the Sy 2 galaxies the best $\chi^{2}$ value for $\cos \theta=0.30$ namely an angle of $73^{\circ}$. The increase of the reflection component is numerically important (2.33 against 1.47 previously) but statistically unconvincing. The reflection is less predominant in the two other Seyfert classes.

(b) Free inclination

We then let the inclination free to avoid as much as possible the dependence between the different parameters. We find that Sy 1.5 galaxies would be seen closer to the normal to the disk with an inclination of $18^{\circ}$ whereas the Sy 1 class would be viewed from an angle of $36^{\circ}$ and the Sy 2 from $74^{\circ}$.

It should be noted that in the PEXRAV implementation of the reflection model, a stronger reflection hump systematically induces a large inclination.

\subsection{Investigating a link between the index slope $\Gamma$ and the reflection parameter $R$}

We investigate whether the index slope $\Gamma$ and the reflection $R$ are independently measured through the "draw" of this possible correlation using XSPEC (Figs. 9 and 10).

This study is performed using an intermediate angle of $\cos \theta=0.45$ for all classes. For both Sy 1 and Sy 2 classes, if the average spectrum has a high index slope (e.g. $\Gamma=2)$ then it has a larger amount of reflection than if it has a hard spectrum (e.g. $\Gamma=1.6$ ). We also note that if any class would have an index slope of less than 1.2 , then reflection is not required to account for the data.

The index slope is the signature of the primary X-ray source. Therefore, a link between the index slope and the reflection amount would indicate that the source of the cooling photons is related to the emission from the material at the origin of the Compton reflection. We will study using individual object spectra whether there is a correlation between $\Gamma$ and $R$ in the Paper II (Deluit \& Courvoisier 2003).

\subsection{Results}

With a model independent method we find that Sy 2 galaxies are similar to Sy 1.5 and differ from Sy 1 galaxies.

With a model dependent method, the best of all theoretical models adopted for the two main classes is the PEXRAV model whereas the Sy 1.5 spectrum is well represented by a broken power law.

Considering the cutoff power law model, $E^{-\Gamma} \exp \left(-E / E_{\mathrm{c}}\right)$, we find that Sy 1.5 and Sy 2 have similar behavior in the sense 


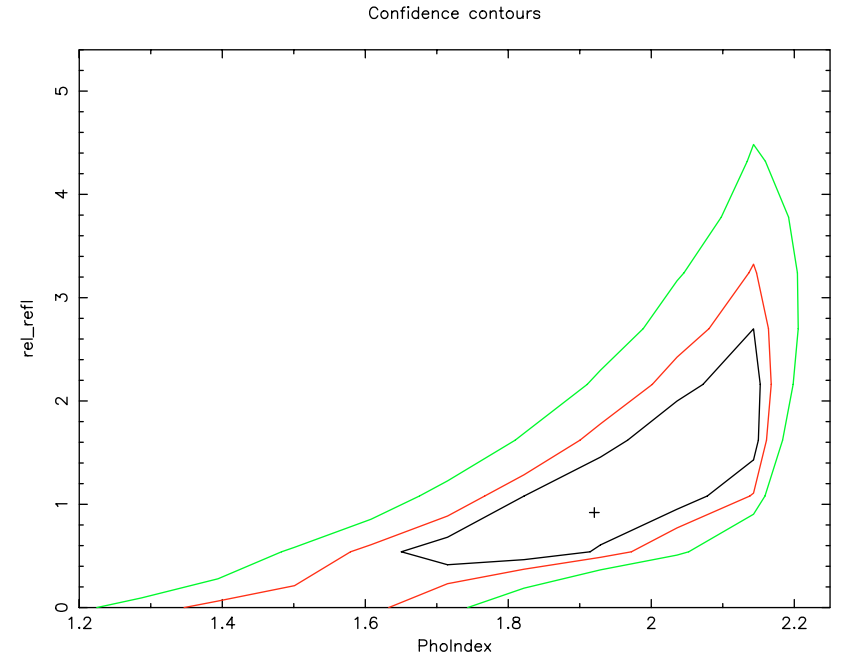

Fig. 9. The 68\%, 90\% and 99\% confidence contours of the index slope $\Gamma$ and the Reflection parameter $R$ for the Sy 1 average spectrum.

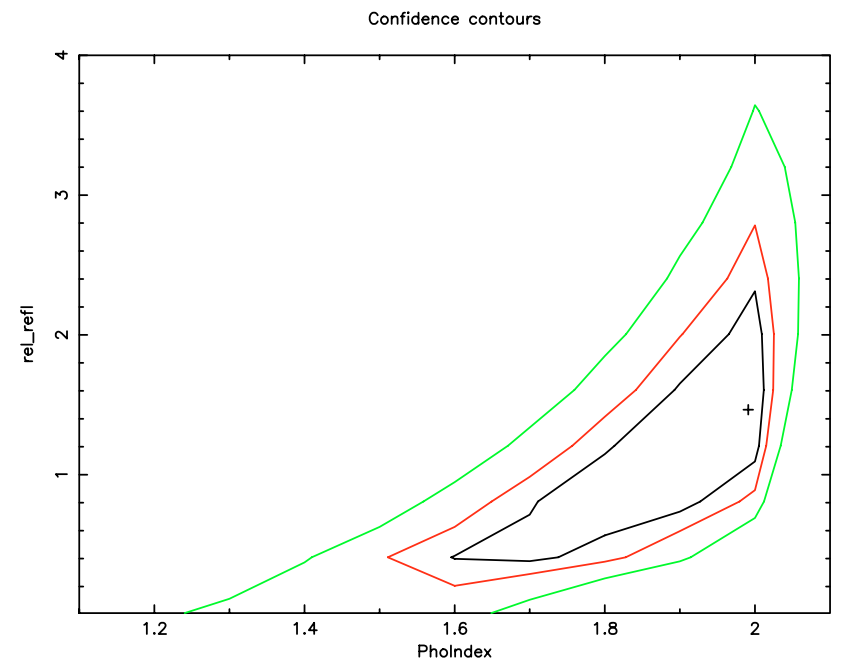

Fig. 10. The $68 \%, 90 \%$ and $99 \%$ confidence contours of the index slope $\Gamma$ and the Reflection parameter $R$ for the Sy 2 average spectrum.

that their spectra do not require any cutoff whereas the Sy 1 spectrum presents a well constrained cutoff at $70_{-17}^{+30} \mathrm{keV}$. In model including reflection, the cutoff energy in Sy 1 emission is found at $221_{-158} \mathrm{keV}$ and still not required in Sy 1.5 and Sy 2 emission.

The main result is thus the presence of a high energy cutoff in Sy 1 that it is absent in other categories. The role and place of Sy 1.5 are not clearly identified. Indeed, Sy 1.5 are close to Sy 1 concerning the relatively low reflection influence, and similar to Sy 2 regarding the absence of a cutoff.

\subsection{Discussion and perspectives}

Our results concerning the index slope of Sy 1 emission confirm the results obtained by Zdziarski et al. (1995) and Gondek et al. (1996) who found $\alpha \sim 0.9$. Our results differ, however, in what regards Sy 2 galaxies. Zdziarski et al. (1995) and Zdziarski et al. (2000) found that Sy 2 are harder than Sy 1 which we do not find. Secondly, Zdziarski et al. (1995) found a negligible reflection in Sy 2 emission whereas in our study, we emphasize the possible importance of this component in the Sy 2 average spectrum.

\subsubsection{High energy cutoff}

The presence or absence of a high energy cutoff is not influenced by the inclination of the system with respect to the line of sight. The measured cutoff in the Sy 1 galaxies emission while none is found in Sy 2 galaxies clearly indicates intrinsic differences in their primary emission.

However this lack could also be explained by a wide distribution of cutoff energies whereas the presence of a cutoff in the Sy 1 average spectrum indicates a narrow range of comptonizing temperatures in the class.

A cutoff in the Sy 1 emission provides, according to thermal Compton models, a reliable estimate of the electron temperature in the Comptonizing medium. The Sy 1 cutoff found at $k_{\mathrm{B}} T=220 \mathrm{keV}$ corresponds to a temperature of $T \sim$ $2.6 \times 10^{9} \mathrm{~K}$ for the electron gas in the case of unsaturated comptonization.

If the absence of a cutoff in Sy 1.5 and Sy 2 galaxies is confirmed, it indicates either the importance of non thermal Compton scattering or a higher temperature of the Comptonizing medium.

\subsubsection{The role of reflection}

We show that Sy 2 galaxies may have a stronger reflection component than the other classes. Our fits also indicate that the inclination of Sy 2 could be larger than that of the other galaxies. Both results are not independent, only models free of this degeneracy will allow us to deconvolve the geometry of the reflection medium from inclination effects.

We also find that the average spectrum of Sy 2 galaxies, more affected by absorption than Sy 1 , shows more reflection than that of Seyfert 1 galaxies suggesting a link between the two processes.

\subsubsection{Implications of our results}

To investigate further these assertions, it is necessary to test our results in a different way, through the study of the distribution of the individual spectral parameters on a large sample of Seyfert galaxies. Therefore, we will study the distribution of the parameters $\Gamma, E_{\mathrm{c}}$ and $R$ for each object of a given class as far as can be measured to date and to compare them in order to test the results found in this paper. This study, presented in Paper II, will allow us to know whether the absence of a high energy cutoff in Sy 1.5 and Sy 2 is due to a wide distribution of values within the class and to study the correlation between the reflection and the power law slope.

Seyfert 2 galaxies have different intrinsic emission and therefore different physical conditions in the inner regions. Sy 2 galaxies in which no cutoff has been found have probably a higher temperature comptonizing medium than Sy 1 galaxies. 
Table A.1. General characteristics and spectral properties of the objects composing the Sy 1 and Sy 1.5 samples.

\begin{tabular}{ccccccc}
\hline \hline Source name & $\begin{array}{c}\text { RA } \\
\text { (h m s) }\end{array}$ & $\begin{array}{c}\text { DEC } \\
\left({ }^{\circ}{ }^{\prime \prime}\right)\end{array}$ & $\begin{array}{c}\text { Redshift } \\
z\end{array}$ & $S / N^{1}$ & $F_{15-136 \mathrm{keV}}{ }^{6}$ & $L_{15-136 \mathrm{keV}^{7}}$ \\
\hline Seyfert 1 galaxies sample & \\
\hline Mrk 335 & 000619.3 & +201210.0 & 0.025 & $3.01 \sigma$ & 1.38 & 1.67 \\
Fairall 9 & 012345.6 & -584814.0 & 0.047 & $11.8 \sigma$ & 5.42 & 23.5 \\
NGC 985 & 023437.6 & -084708.0 & 0.043 & $9.69 \sigma$ & 3.63 & 13.1 \\
1H0419-577 & 042601.0 & -571200.0 & 0.104 & $2.00 \sigma$ & 1.18 & 25.7 \\
Mrk 110 & 092512.9 & +521711.0 & 0.035 & $12.8 \sigma$ & 4.35 & 10.4 \\
REJ1034+393 & 103438.8 & +393834.0 & 0.042 & $2.14 \sigma$ & 0.71 & 2.44 \\
NGC 3783 & 113901.7 & -374420.0 & 0.011 & $48.5 \sigma$ & 14.2 & 3.31 \\
NGC 4051 & 120309.5 & +443150.9 & 0.002 & $7.25 \sigma$ & 2.06 & 0.02 \\
NGC 4593 & 123939.4 & -052038.0 & 0.008 & $23.0 \sigma$ & 9.63 & 1.19 \\
MCG 6-30-15 & 133553.2 & -341748.0 & 0.008 & $33.0 \sigma$ & 7.19 & 0.84 \\
IC 4329A & 134919.2 & -301835.9 & 0.016 & $99.0 \sigma$ & 22.6 & 11.2 \\
ESO 141-G55 & 192114.3 & -584013.0 & 0.036 & $15.3 \sigma$ & 5.37 & 13.6 \\
Mrk 509 & 204409.7 & -104323.9 & 0.034 & $27.4 \sigma$ & 9.73 & 21.9 \\
NGC 7469 & 230315.5 & +085226.0 & 0.016 & $28.0 \sigma$ & 5.11 & 2.52 \\
\hline \multicolumn{7}{c}{ Seyfert 1.5 galaxies sample } \\
\hline Mrk 1152 & 011350.2 & -145059.9 & 0.053 & $3.32 \sigma$ & 0.59 & 3.22 \\
NGC 526A & 012354.1 & -350356.0 & 0.019 & $13.7 \sigma$ & 5.89 & 4.15 \\
MCG 8-11-11 & 055453.5 & +462621.0 & 0.021 & $29.3 \sigma$ & 11.4 & 9.25 \\
Mrk 6 & 065212.1 & +742536.9 & 0.019 & $21.0 \sigma$ & 7.67 & 5.24 \\
NGC 3516 & 110647.2 & +723408.0 & 0.009 & $29.8 \sigma$ & 11.5 & 1.71 \\
Mrk 766/NGC 4253 & 121826.2 & +294846.0 & 0.013 & $14.0 \sigma$ & 3.63 & 1.16 \\
NGC 5548 & 1417595 & +250812.0 & 0.017 & $52.6 \sigma$ & 10.6 & 6.06 \\
Mrk 841 & 150401.2 & +102616.0 & 0.036 & $7.71 \sigma$ & 2.71 & 6.92 \\
NGC 7213 & 220916.2 & -471000.0 & 0.006 & $31.4 \sigma$ & 7.03 & 0.49 \\
\hline
\end{tabular}

Note:

${ }^{1}$ Integrated signal to noise ratio in the PDS energy range.

22 available observations.

${ }^{3} 3$ available observations.

${ }^{4} 4$ available observations.

5 available observations.

${ }^{6}$ Integrated flux in $10^{-11} \mathrm{erg} \mathrm{s}^{-1} \mathrm{~cm}^{-2}$.

${ }^{7}$ Integrated luminosity in $10^{43} \mathrm{erg} \mathrm{s}^{-1}$.

This reveals that the ratio heating/cooling of this medium is different in both classes.

Our study has to be pursued within the Sy 2 galaxies where two subclasses known as Sy 2 with Polarized Broad Lines (PBL) and without PBL are found. Unified schemes predict that both types have a hidden Sy 1 nucleus. We have to search for common behavior in the intrinsic emission of both Sy 2 subclasses and to compare them with Sy 1 emission features (see Deluit 2003).

The nature of the fueling mechanism occurring in the nucleus is unsolved and could also contribute to the Seyfert 2 particularity. Indeed, Sy 2 galaxies have been found to host starbursts more often than type 1 AGNs (Levenson et al. 2001). We could expect that in Sy 2 objects the accreted matter originates predominantly in starbursts.

\section{Conclusion}

We obtained and studied the average hard X-ray spectra of Seyfert 1, 1.5 and 2 galaxies observed by the PDS on board BeppoSAX. We emphasized several differences in the central engine behavior of the two main Seyfert classes indicating that the Seyfert 1 and 2 galaxies have at least various physical conditions in their inner regions or are different objects. The differences cannot be due only to inclination/absorption effects as claimed by unified models.

The next steps in the Seyfert studies will be the search for the presence of a high energy cutoff in individual Seyfert galaxies to investigate the importance of the different physical processes in their emission (thermal and/or non thermal) and the investigation of the nature of the reflection medium (disk or material surrounding the nucleus), of its composition (density, homogeneity) and its localization. It is also important in a different set of considerations to investigate whether Seyfert 1 and Seyfert 2 have intrinsic differences in their star formation rate, morphology and/or environment.

\section{Appendix A: The final sample of the objects studied}

We present below the characteristics of the objects composing the final sample of our study. Table A.1 includes the Seyfert 1 
Table A.2. General characteristics and spectral properties of the objects composing the Sy 2 sample.

\begin{tabular}{|c|c|c|c|c|c|c|c|}
\hline Source name & $\begin{array}{c}\mathrm{RA} \\
(\mathrm{h} \mathrm{m} \mathrm{s})\end{array}$ & $\begin{array}{l}\text { DEC } \\
\left({ }^{\circ}, \prime\right)\end{array}$ & $\begin{array}{c}\text { Redshift } \\
z\end{array}$ & $N_{\mathrm{H}}^{1}$ & $S / N^{4}$ & $F_{15-136 \mathrm{kev}}{ }^{5}$ & $L_{15-136 \mathrm{kev}}{ }^{6}$ \\
\hline IRAS 00198-7926 & 002153.8 & -791008.0 & 0.073 & - & $2.10 \sigma$ & 2.18 & 22.9 \\
\hline NGC 1358 & 033339.5 & -050520.0 & 0.013 & - & $2.28 \sigma$ & 1.38 & 0.48 \\
\hline IRAS 05189-2524 & 052101.3 & -252142.9 & 0.043 & $4.90^{[B]}$ & $2.66 \sigma$ & 1.14 & 4.04 \\
\hline NGC 2110 & 055211.2 & -072720.8 & 0.008 & $2.89^{[H]}$ & $20.5 \sigma$ & 8.62 & 1.01 \\
\hline NGC $2992^{2}$ & 094542.0 & -141936.9 & 0.008 & $0.69^{[W 96]}$ & $25.8 \sigma$ & 7.98 & 0.91 \\
\hline MCG 5-23-16 & 094740.1 & -305653.9 & 0.008 & $1.62^{[\mathrm{W} 97]}$ & $45.8 \sigma$ & 19.8 & 2.62 \\
\hline IRAS F12072-0444 & 120945.1 & -050114.9 & 0.128 & $0.17^{[D]}$ & $4.88 \sigma$ & 1.84 & 61.5 \\
\hline NGC $4388^{2}$ & 122546.7 & +123944.0 & 0.008 & $42.0^{[B]}$ & $63.8 \sigma$ & 22.1 & 3.00 \\
\hline NGC $4507^{3}$ & 123537.0 & -395432.0 & 0.012 & $29.2^{[C]}$ & $58.1 \sigma$ & 19.1 & 5.12 \\
\hline NGC 4939 & 130414.5 & -102026.9 & 0.010 & $30.0^{[M]}$ & $3.56 \sigma$ & 2.26 & 0.47 \\
\hline NGC 5252 & 133816.3 & +043220.0 & 0.023 & $4.33^{[C a]}$ & $2.86 \sigma$ & 2.26 & 2.34 \\
\hline NGC $5506^{3}$ & 141314.8 & -031228.0 & 0.006 & $3.40^{[s]}$ & $69.0 \sigma$ & 17.0 & 1.25 \\
\hline NGC 5674 & 143352.0 & +052728.0 & 0.025 & $7.00^{[B]}$ & $5.05 \sigma$ & 1.69 & 2.07 \\
\hline NGC 6300 & 171700.3 & -624915.0 & 0.004 & $60.0^{[L e]}$ & $23.1 \sigma$ & 8.35 & 0.22 \\
\hline IRAS 18325-5926/F 49 & 183658.3 & -592409.0 & 0.020 & $1.26^{[B]}$ & $6.06 \sigma$ & 1.25 & 0.99 \\
\hline ESO 103-G35 & 183820.3 & -652540.0 & 0.013 & $15.9^{[T]}$ & $7.84 \sigma$ & 7.96 & 2.71 \\
\hline IRAS $20210+1121$ & 202325.6 & +113132.9 & 0.056 & $\leq 6.0^{[U]}$ & $2.24 \sigma$ & 0.87 & 5.45 \\
\hline NGC $7172^{2}$ & 220202.2 & -315212.0 & 0.009 & $8.61^{[G]}$ & $9.73 \sigma$ & 4.43 & 0.65 \\
\hline NGC 7314 & 223546.0 & -260301.0 & 0.005 & $1.16^{[T]}$ & $18.5 \sigma$ & 7.07 & 0.30 \\
\hline IRAS $23060+0505$ & 230833.8 & +052129.0 & 0.173 & $8.40^{[B r]}$ & $4.20 \sigma$ & 1.79 & 112 \\
\hline NGC 7582 & 231823.5 & -422211.9 & 0.005 & $12.4^{[X]}$ & $24.2 \sigma$ & 10.5 & 0.57 \\
\hline NGC 7679 & 232846.8 & +033038.9 & 0.017 & $0.04^{[D C]}$ & $4.98 \sigma$ & 1.36 & 0.76 \\
\hline
\end{tabular}

Note:

References concerning the hydrogen column density:

$B$ : Bassani et al. (1999), Br: Brandt et al. (1997), Ca: Cappi et al. (1996), Co: Comastri et al. (1998), D: Deluit, this work, see Paper II of this study, DC: Della Ceca et al. (2001), G: Guainazzi et al. (1998), H: Hayashi et al. (1996), Le: Leighly et al. (2000), M: Maiolino et al. (1998), $S$ : Smith \& Done (1996), $T$ : Turner et al. (1997), $U$ : Ueno et al. (1998), W96: Weaver et al. (1996), W97: Weaver et al. (1997), X: Xue et al. (1998).

${ }^{1}$ Hydrogen column density in $10^{22} \mathrm{~cm}^{-2}$ units.

2 available observations.

3 available observations.

${ }^{4}$ Integrated signal to noise ratio.

${ }^{5}$ Integrated flux in $10^{-11} \mathrm{erg} \mathrm{s}^{-1} \mathrm{~cm}^{-2}$.

${ }^{6}$ Integrated Luminosity in $10^{43} \mathrm{erg} \mathrm{s}^{-1}$.

and Seyfert 1.5 galaxies subsamples and Table A.2 represents the Seyfert 2 subsample.

\section{Appendix B: The role of absorption in Seyfert 2 class studies}

According to actual models, the X-ray emission of many Seyfert 2 galaxies is characterized by a power law similar to that observed in Seyfert 1, with a cutoff at low energies $(<10 \mathrm{keV})$ due to absorption effects produced by gas column densities higher than $10^{22} \mathrm{~cm}^{-2}$. Seyfert 2 galaxies show a broad range of absorption, with column densities varying from $\leq 10^{22} \mathrm{~cm}^{-2}$ to $\geq 10^{25} \mathrm{~cm}^{-2}$ (Risaliti et al. 1999). These results yield to a further classification among Seyfert 2 galaxies: Compton thin and Compton thick sources depending on whether their column density is respectively lower or larger than the inverse Thomson cross-section $N_{\mathrm{H}}=\sigma_{\mathrm{T}}^{-1}=1.5 \times$ $10^{24} \mathrm{~cm}^{-2}$.

Therefore, for objects presenting a column density less than $1.5 \times 10^{24} \mathrm{~cm}^{-2}$ (Compton thin), high energy X-rays penetrate the absorbing material, making the nuclear source visible to the observer and allowing him to measure the hydrogen column density. For objects with a column density higher than $1.5 \times 10^{24} \mathrm{~cm}^{-2}$ (Compton thick), the matter is optically thick to the Compton scattering and the nucleus is hardly visible. In the last few years, BeppoSAX data revealed a new highly absorbed class with only a lower limit on the column density $N_{\mathrm{H}}>10^{25} \mathrm{~cm}^{-2}$ (Risaliti et al. 1999), no detection of the intrinsic emission can be expected at energies between 15 and $150 \mathrm{keV}$.

The photoelectric absorption cross section as a function of energy is well described by Morrison \& McCammon (1983) up to $10 \mathrm{keV}$. The component due to Compton scattering is described by the Thomson cross section equal to $6.65 \times 10^{-25} \mathrm{~cm}^{-2}$. Compton scattering plays an important role with the photoelectric cross section above $4 \mathrm{keV}$, and dominates above $10 \mathrm{keV}$. Starting with the analytic formulae Morrison \& McCammon (1983)

$\sigma_{\text {photoelectric }}=\left(C_{0}+C_{1} E+C_{2} E^{2}\right) E^{-3} \times 10^{-24} \mathrm{~cm}^{-2}$, where $C_{0}=701.2, C_{1}=25.2$ and $C_{2}=0$. 

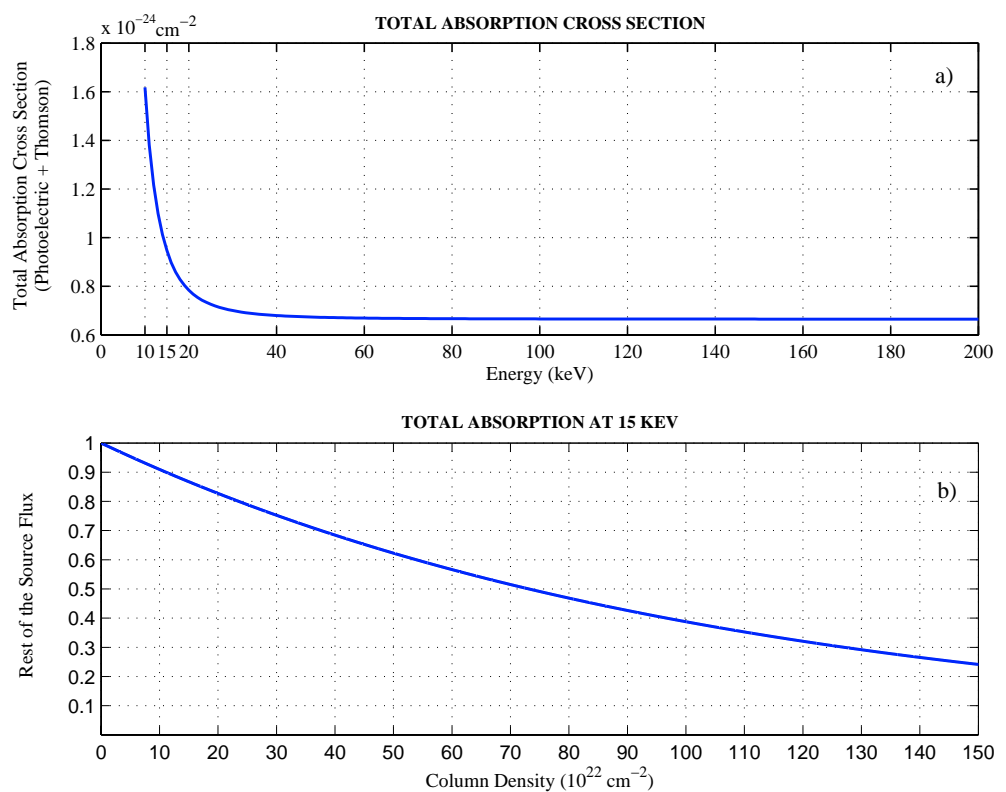

Fig. B.1. Value and influence of the absorption at high energies. a) gives the value of the total absorption cross section composed of the photoelectric and Thomson components. b) gives the fraction of the rest flux at $15 \mathrm{keV}$ as a function of the column density.

The photoelectric cross section at $10 \mathrm{keV}$ is

$\sigma_{\mathrm{ph}}(10 \mathrm{keV})=0.9532 \times 10^{-24} \mathrm{~cm}^{-2}$.

At $10 \mathrm{keV}$ the $E^{-3}$ dependence is dominant, we thus extrapolate B.1. to higher energies using

$\sigma_{\mathrm{ph}}($ Energy $)=\sigma_{\mathrm{ph}}(10 \mathrm{keV}) \times\left(\frac{\text { Energy }}{10 \mathrm{keV}}\right)^{-3}$.

We present in the upper panel of Fig. B.1. the value of the total absorption cross section as a function of energy. The total absorption of the emission from the central engine is characterized as

Flux $_{\text {observed }}=$ Flux $x_{\text {source }} \times \mathrm{e}^{-\sigma_{\mathrm{Th}} N_{\mathrm{H}}} \times \mathrm{e}^{-\sigma_{\mathrm{ph}}(E) N_{\mathrm{H}}}$.

We compute this at $15 \mathrm{keV}$, the lower energy used in our study, and show the result in the lower panel of the Fig. B.1. An object is considered as strongly absorbed when its flux has decreased by $50 \%$ due to absorption effects. We see in the lower panel of Fig. B.1 that this value corresponds to a column density around $70 \times 10^{22} \mathrm{~cm}^{-2}$.

Therefore, we consider that below a column density of $70 \times 10^{22} \mathrm{~cm}^{-2}$ the absorption is not an important effect at the energies covered in this study. Thus, we excluded objects with higher hydrogen column density.

\section{Appendix C: Average counts spectra fitted by the different models}

We present below the counts spectrum of each class fitted by the different models applied in this study.

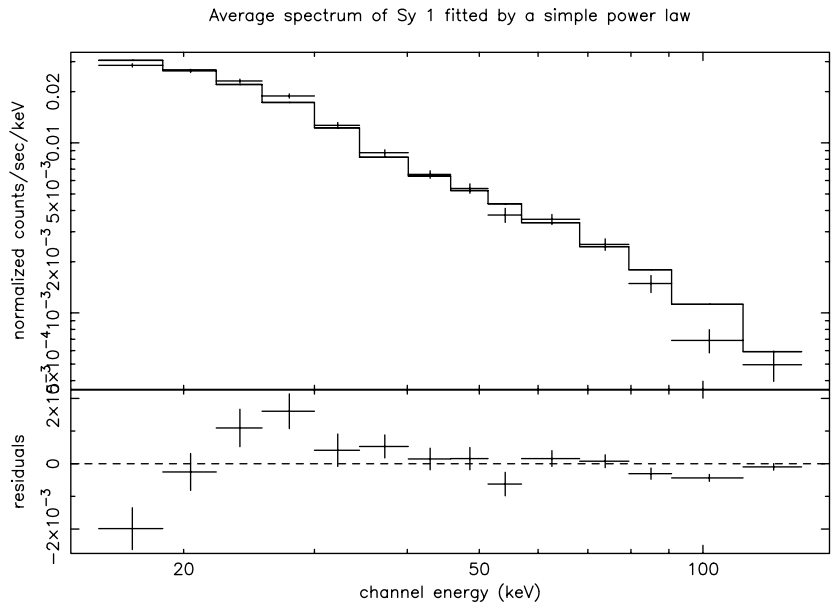

Fig. C.1. Sy 1 average spectrum fitted by a power law model and residuals.

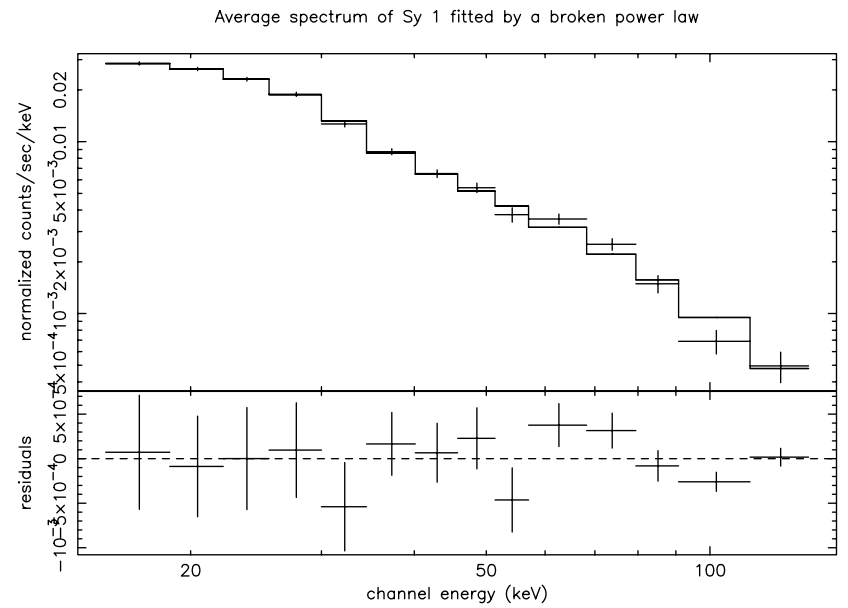

Fig. C.2. Sy 1 average spectrum fitted by a broken power law model and residuals. 


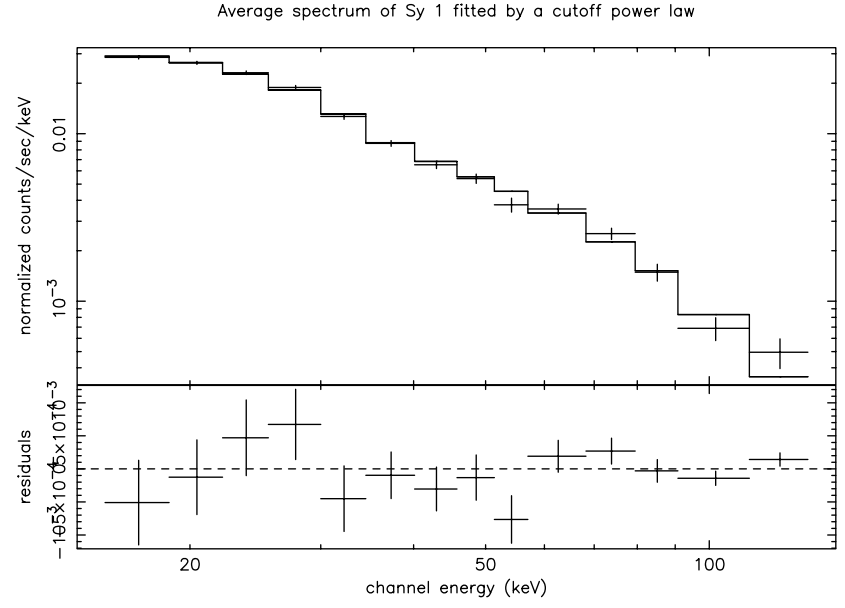

Fig. C.3. Sy 1 average spectrum fitted by a cutoff power law model and residuals.

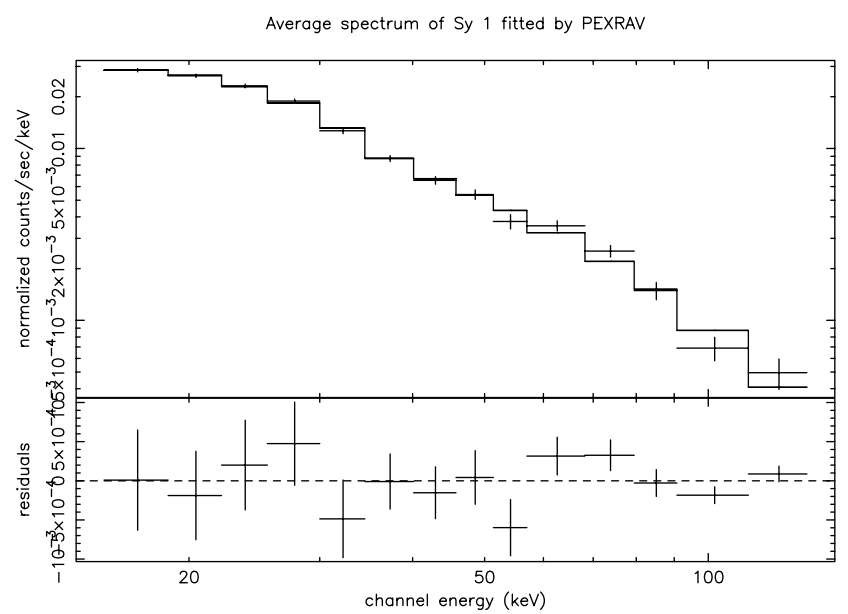

Fig. C.4. Sy 1 average spectrum fitted by the PEXRAV model $(\cos \theta=$ 0.45 ) and residuals.

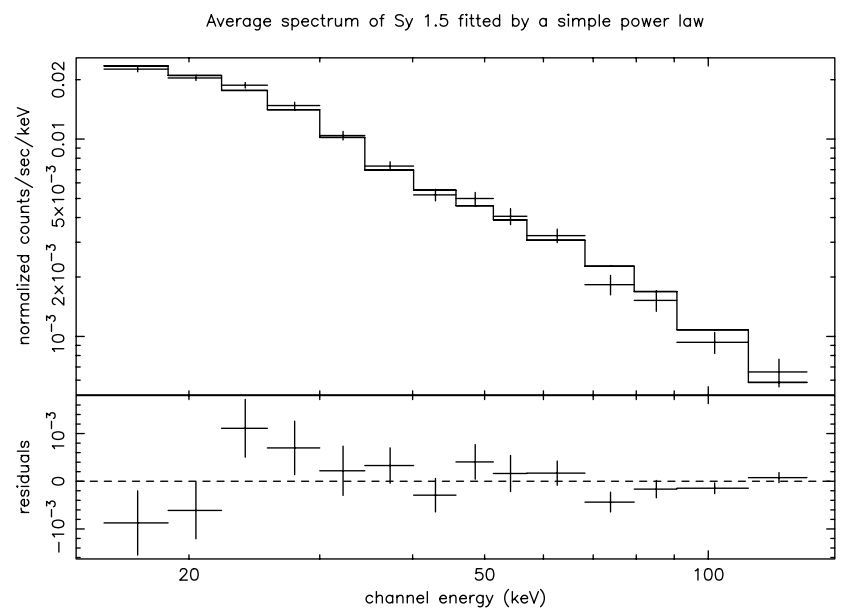

Fig. C.5. Sy 1.5 average spectrum fitted by a power law model and residuals.

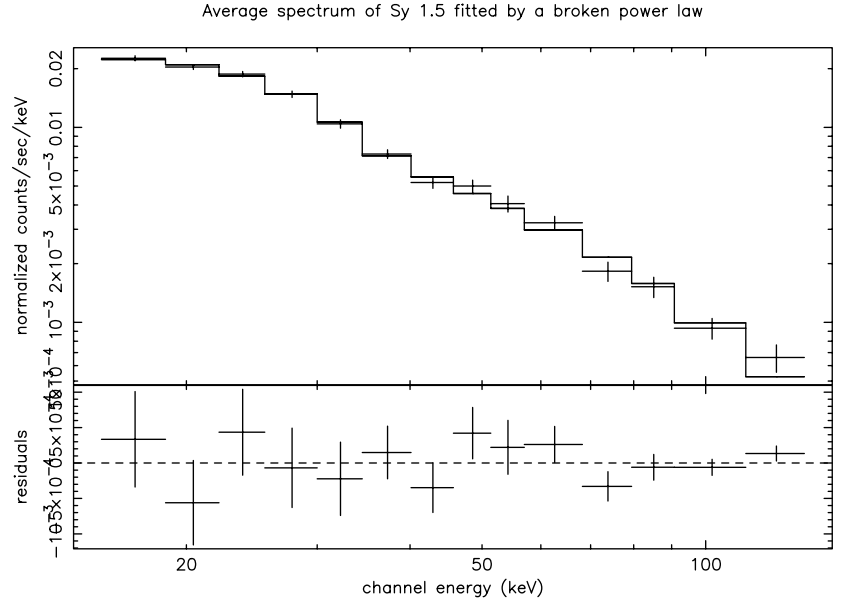

Fig. C.6. Sy 1.5 average spectrum fitted by a broken power law model and residuals.

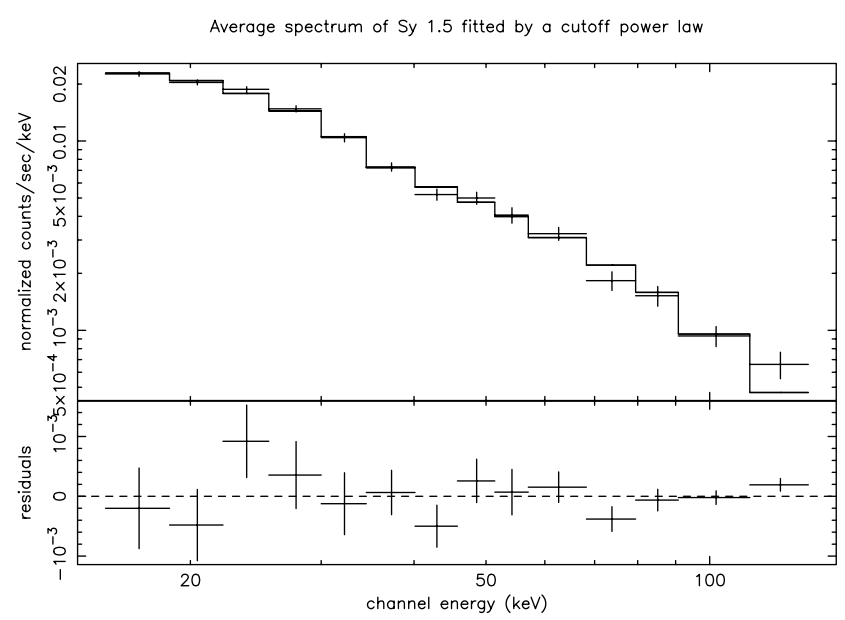

Fig. C.7. Sy 1.5 average spectrum fitted by a cutoff power law model and residuals.

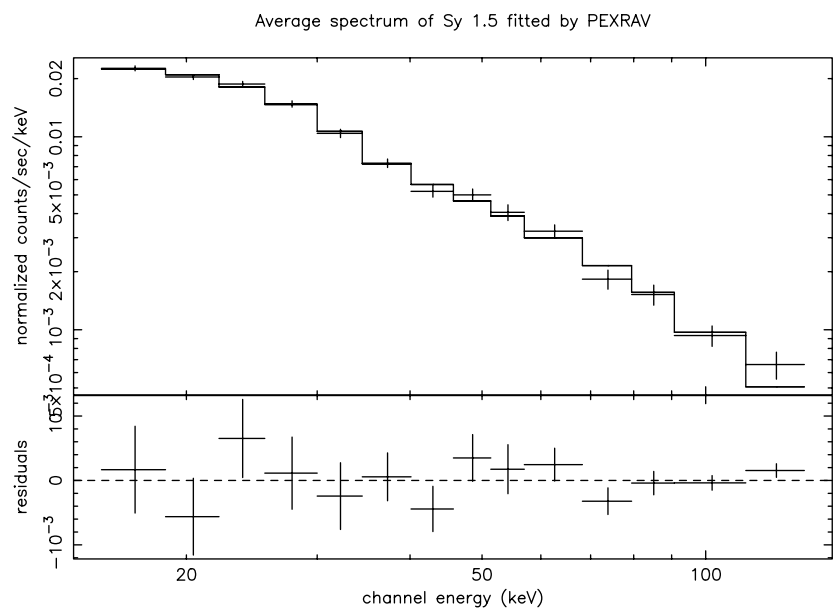

Fig. C.8. Sy 1.5 average spectrum fitted by the PEXRAV model $(\cos \theta=0.45)$ and residuals. 


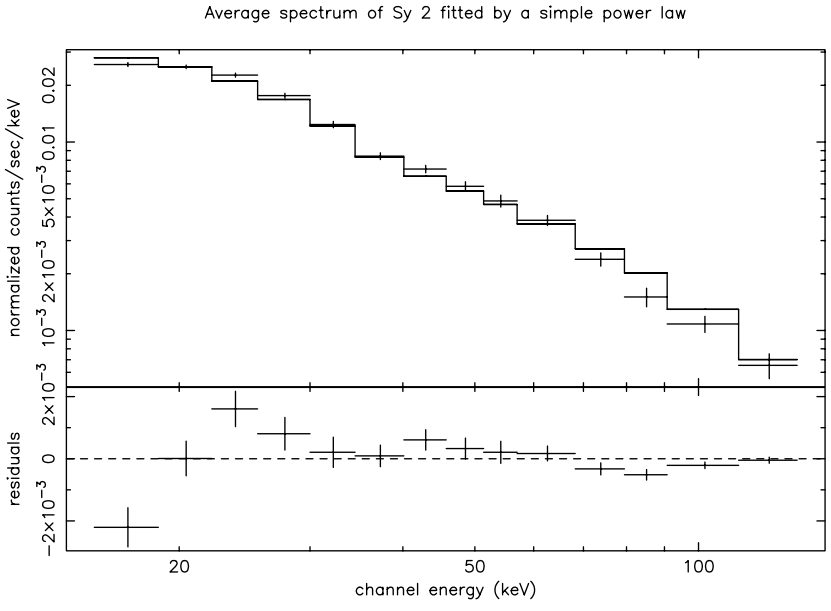

Fig. C.9. Sy 2 average spectrum fitted by a power law model and residuals.

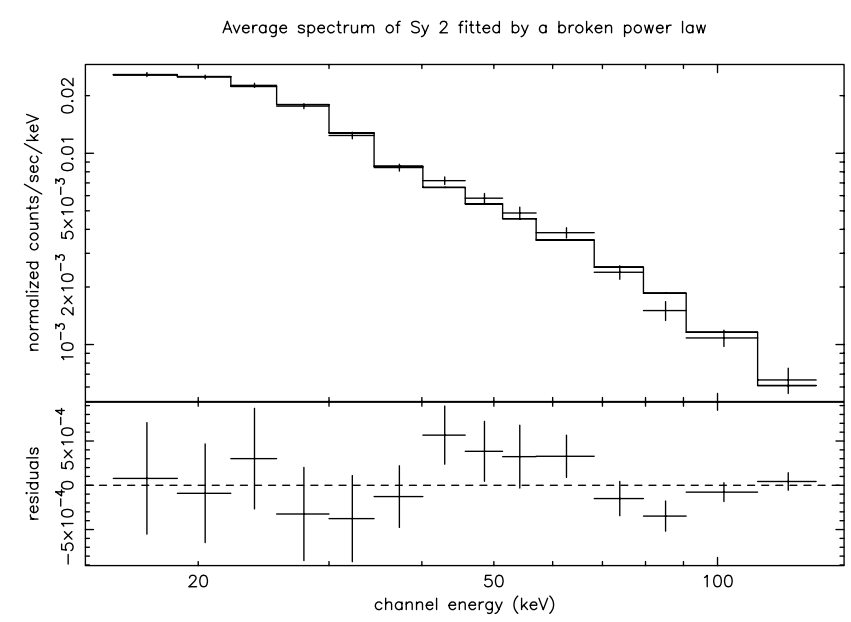

Fig. C.10. Sy 2 average spectrum fitted by a broken power law model and residuals.

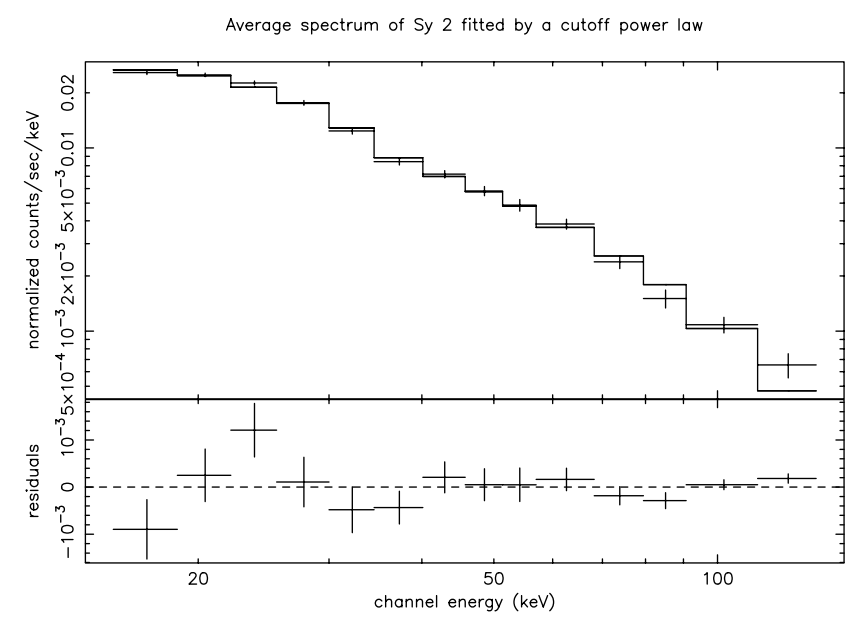

Fig. C.11. Sy 2 average spectrum fitted by a cutoff power law model and residuals.

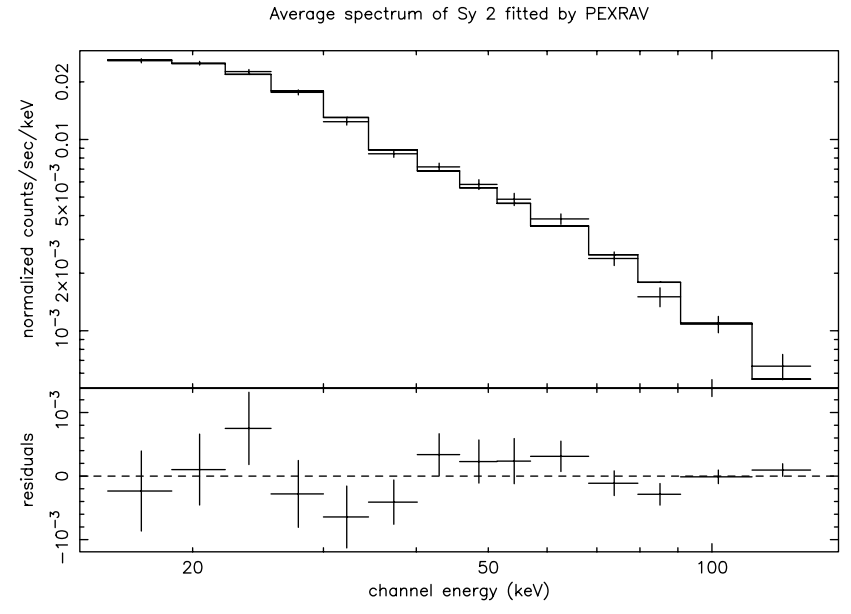

Fig. C.12. Sy 2 average spectrum fitted by the PEXRAV model $(\cos \theta=0.45)$ and residuals.

\section{References}

Alexander, D. M. 2001, MNRAS, 320, L15

Antonucci, R. R. J., \& Miller, J. S. 1985, ApJ, 297, 621

Arnaud, K. A. 1996, in ASP Conf. Ser., 101, Astronomical Data Analysis Software and Systems V, 5, 17

Awaki, H., Ueno, S., Taniguchi, Y., \& Weaver, K. A. 2000, ApJ, 542, 175

Bassani, L., Dadina, M., Maiolino, R., et al. 1999, ApJS, 121, 473

Boella, G., Chiappetti, L., Conti, G., et al. 1997, A\&AS, 122, 327

Brandt, W. N., Fabian, A. C., Takahashi, K., et al. 1997, MNRAS, 290, 617

Cappi, M., Mihara, T., Matsuoka, M., et al. 1996, ApJ, 456, 141

Comastri, A., Vignali, C., Cappi, M., et al. 1998, MNRAS, 295, 443

Della Ceca, R., Pellegrini, S., Bassani, L., et al. 2001, A\&A, 375, 781

Deluit, S. 2003, in preparation

Deluit, S., \& Courvoisier, T. J.-L. 2003, in preparation

Frontera, F., Costa, E., dal Fiume, D., et al. 1997, A\&AS, 122, 357

Gondek, D., Zdziarski, A. A., Johnson, W. N., et al. 1996, MNRAS, 282,646

Gu, Q., Maiolino, R., \& Dultzin-Hacyan, D. 2001, A\&A, 366, 765

Guainazzi, M., Fiore, F., Matt, G., \& Perola, G. C. 2001, MNRAS, 327,323

Guainazzi, M., Matt, G., Antonelli, L. A., et al. 1998, MNRAS, 298, 824

Hayashi, I., Koyama, K., Awaki, H., \& Yamauchi, S. U. S. 1996, PASJ, 48,219

Lampton, M., Margon, B., \& Bowyer, S. 1976, ApJ, 208, 177

Leighly, K. M., Cappi, M., Kay, L., et al. 2000, in Rossi 2000: Astrophysics with the Rossi X-ray Timing Explorer. March 2224, 2000 at NASA's Goddard Space Flight Center, Greenbelt, MD USA, E125

Levenson, N. A., Weaver, K. A., \& Heckman, T. M. 2001, ApJ, 550, 230

Lumsden, S. L., Heisler, C. A., Bailey, J. A., Hough, J. H., \& Young, S. 2001, MNRAS, 327, 459

Magdziarz, P., \& Zdziarski, A. A. 1995, MNRAS, 273, 837

Maiolino, R., Salvati, M., Bassani, L., et al. 1998, A\&A, 338, 781

Miller, J. S., \& Goodrich, R. W. 1990, ApJ, 355, 456

Moran, E. C., Barth, A. J., Kay, L. E., \& Filippenko, A. V. 2000, ApJ, $540, \mathrm{~L} 73$

Morrison, R., \& McCammon, D. 1983, ApJ, 270, 119

Nandra, K., George, I. M., Mushotzky, R. F., Turner, T. J., \& Yaqoob, T. 1997, ApJ, 477, 602 
Nandra, K., \& Pounds, K. A. 1994, MNRAS, 268, 405

Parmar, A. N., Martin, D. D. E., Bavdaz, M., et al. 1997, A\&AS, 122, 309

Risaliti, G., Maiolino, R., \& Salvati, M. 1999, ApJ, 522, 157

Rybicki, G. B., \& Lightman, A. P. 1979, Radiative processes in astrophysics (New York, Wiley-Interscience), 393

Smith, D. A., \& Done, C. 1996, MNRAS, 280, 355

Tadhunter, C., \& Tsvetanov, Z. 1989, Nature, 341, 422

Tran, H. 1995, ApJ, 440, 565

Turner, T. J., George, I. M., Nandra, K., \& Mushotzky, R. F. 1997, ApJ, 488, 164

Ueno, S., Ward, M. J., O’Brien, P. T., Stirpe, G. M., \& Matt, G. 1998, in The Active X-ray Sky: Results from BeppoSAX and RXTE, 554
Weaver, K. A., Nousek, J., Yaqoob, T., et al. 1996, ApJ, 458, 160

Weaver, K. A., Yaqoob, T., Mushotzky, R. F., et al. 1997, ApJ, 474, 675

Xue, S., Otani, C., Mihara, T., Cappi, M., \& Matsuoka, M. 1998, PASJ, 50, 519

Young, S., Hough, J. H., Efstathiou, A., et al. 1996, MNRAS, 279, L72

Zdziarski, A. A., Johnson, W. N., Done, C., Smith, D., \& McNaronBrown, K. 1995, ApJ, 438, L63

Zdziarski, A. A., Lubinski, P., \& Smith, D. A. 1999, MNRAS, 303, L11

Zdziarski, A. A., Poutanen, J., \& Johnson, W. N. 2000, ApJ, 542, 703 